\title{
ENHANCEMENT OF SURFACE-ATMOSPHERE FLUXES BY DESERT-FRINGE VEGETATION THROUGH REDUCTION OF SURFACE ALBEDO AND OF SOIL HEAT FLUX
}

J. Otterman

Tel Aviv University, Ramat Aviv, Israel

Current Address:

NASA/Goddard Space Flight Center

Code 623

Greenbelt, MD 20771

(MASA-CR-181442) EBEANCBAENT OF SURPACB-ATHOSPRERE FL OXES BY DESERT-FRIIGE VEGETATION THROOGH REDUCTIOR OF SURFACE ALBEDO ALD OF SOIL HEAT FLUX STE1-AViv Univ. 130 p Avail: NTIS HC A03/MP 101
M8-11231

Onclas G3/46 0105444 


\section{ABSTRACT}

Under the arid conditions prevailing at the end of the dry season (near the Autumn equinox) in the western Negev/ northern Sinai region, vegetation causes a sharp increase relative to bare soil in the daytime sensible heat flux from the surface to the atmosphere. Two distinct mechanisms are involved: the increase in the surface abosorptivity (co-albedo) and a decrease (as compared to bare soil with the same absorption of insolation) in the surface (soil/plants) heat flux. Based on the values of the soil albedo and of the plant density in a fenced area (an exclosure) in the sinai, the increase in the surface absorptivity by vegetation fully protected from grazing is by a factor $E_{d a}$ of 1.23 at solar zenith angle of $30^{\circ}$ and by a factor of 1.33 at solar zenith angle of $60^{\circ}$. Neglecting the latent heat flux, which corresponds to less than $0.5 \mathrm{~mm}$ of water evaporated per day, this mechanism increases the sensible heat flux in the same ratios. These desertfringe plants, mainly thin, predominantly vertical grey/brown twigs and stalks, readily transfer the absorbed solar radiation to the atmosphere. The intimate aerodynamic contact between the plants and the air flow can be inferred from the fact that the plant temperatures are relatively close to the air temperatures. The heat storage in the plants is considered negligible. The soil heat flux is significantly reduced when compared to that into bare soil with the same surface albedo as the plants/soil surface. Assuming that for the bare soil the daytime soil heat flux is half of the net radiation (from measurements by Clothier et al., 1986), this soil heat flux reduction translates according to our model into a daytime enhancement $\mathbf{E}_{\mathrm{dg}}$ of the sensible heat flux by a factor of 1.27 at solar zenith angle of $30^{\circ}$, and by a factor of 1.47 at solar zenith angle of $60^{\circ}$. The combined effect of the plants, $E_{\mathrm{da}} \cdot E_{\mathrm{dg}}$ ' by both the albedo and the soil heat reduction can thus 
increase the sensible heat flux by a factor of 1.56 at solar zenith angle of $30^{\circ}$ and by a factor of 1.95 at solar zenith angle of $60^{\circ}$. These calculations are for the direct solar beam only; a less steep increase with solar zenith angle occurs when the scattered radiation is considered.

By increasing the sensible heat flux to the atmosphere through the albedo and the soil heat flux reductions, the desert-fringe vegetation increases the daytime convection and the growth of planetary boundary layer. When moisture moves from outside the dry region, probabilities of precipitation are then enhanced. The effects can be expected to be especially large during the morning and afternoon hours, that is, at moderately large solar zenith angles. Conversely, removal of vegetation by overgrazing, by reducing the sensible heat flux, tends to reduce daytime convective precipitation, producing higher probabilities of drought conditions.

This assessment of overgrazing (or conversely, of protecting the vegetation over a large region) is based on observations in the Sinai/Negev, where the soil albedo is high (about 0.42 ) and where overgrazing produces an essentially bare soil. The calculations should not be automatically applied to all arid regions. The effects would be smaller when calculated for soils with a lower albedo. In the Kalahari and in northern Mexico, bushes or trees remain in the overgrazed areas, and strong climatic effects of overgrazing cannot be postulated. Even if our assessment for sinai/Negev does not quantitatively apply throughout Africa, the current practice in many African countries of maintaining a large population of grazing animals, can contribute through the mesoscale mechanisms described here to reduced daytime convective precipitation, perpetuating higher probabilities of drought. Time-of-day analysis of precipitation in Africa appears worthwhile, to better assess the role of the surface conditions in contributing to drought. 


\section{Introduction}

During the last fifteen years, vast regions of arid and sub-arid Africa have experienced incidents of severe drought. While drought in these regions had been recorded in the past (Nicholson, 1979), the recent persistence of drought is puzzling. The statistics of precipitation are such that the failure of only one or two intense rainstorms to materialize in a given year signifies a drought year (S. Nicholson, personal communication).

Addressing this problem area, we discuss here how denuding the surface of its natural vegetation can reduce the convection and thus reduce the probabilities of convective precipitation. This study is aimed to be applicable to the conditions prevailing at the end of the dry season (September/October) in northern Sinai/western Negev. The analysis is based on measurements by Idso et al. (1975) and Clothier et al. (1986) of the net radiation and the soil heat flux; on a simple model of absorption of solar radiation by the plants/soil surface, developed specifically for the desertscrub growing on the extremely bright soil of the northern sinai (Otterman 1981a); and on interpretation of ground measurements and satellite data over an exclosure in this region (Otterman 1981b; Otterman and Robinove, 1982; Otterman and Tucker, 1985).

The difference between the net radiation balance $R_{n}$ and the soil heat flux G, measured and discussed by Idso et al. (1975), constitutes the primary term for evaluating the potential evaporation from which the actual evaporation (the latent heat flux from the surface to the atmosphere) can be assessed under a set of assumptions or measured conditions. In our analysis we apply the data obtained by Idso et al. (1975) to a different purpose, to 
assess the sensible heat flux from a dry surface. It is thus appropriate to discuss first the climatological circumstances that prevail in Sinai/Negev at the Autumn equinox, before the first rain, which justify this approach.

The Negev and the northern Sinai lie at the fringe of the great desert belt. Based on changes in the distribution pattern of various woody species. during the last 5000 years, the climate of this region has apparently become drier and warmer (Waisel, 1986). The rains in Sinai/Negev come predominantly during the four winter months, November - February, with precipitation ranging from $100 \mathrm{~mm} \mathrm{y}^{-1}$ in the northern Sinai to $200 \mathrm{~mm} y^{-1}$ in the western Negev (to the north of the northern Sinai). Dew in the sumer possibly can contribute $20 \mathrm{~mm} \mathrm{y}^{-1}$ of additional moisture. Considering strong run-off, no more than $120 \mathrm{~mm}$ is available for evaporation/evapotranspiration during the eight dry months March - October, that is, no more that $0.5 \mathrm{~mm} /$ day as the average. Toward the end of the dry season the evaporation should be less than this average figure. Analyzing in our study the differences between vegetated and denuded areas, we focus on the ratio of the sensible heat flux from a vegetated surface to that from a bare soil. The latent heat flux from either surface is neglected. The calculated ratios apply more accurately to the combined sensible and latent heat fluxes. If we assumed the evaporation rate for each surface as $0.5 \mathrm{~mm}^{\mathrm{day}}{ }^{-1}$, the enhancement ratios for the sensible heat flux by the plants would be slightly higher than the ratios reported here.

our aim in this study is to treat in a realistic manner the absorption of the solar radiation by a vegetated surface in a desert-fringe region and the transfer to the atmosphere of the sensible heat. Daylong measurements of the radiation temperature of desert scrub and of soil in the sinai exclosure (Otterman, 1981a) clearly indicate that the temperatures of these two surface 
components as a function of time of day follow distinct, though similar, patterns. Both components show radiation temperatures rising towards noon and declining in the afternoon. However, these plants, predominantly dry twigs and stems protruding approximately vertically from the bright soil (for a photograph of the exclosure, see Otterman and Tucker, 1985) intercept most of the direct solar beam when the sun is near the horizon, and therefore in the early morning warm up more rapidly than the soil. Later in the morning, the soil warms up more steeply, at which time the plant radiation temperatures become lower than those of the soil, closer to the air temperatures (Otterman, 1981a)

These measurements suggest that we analyze the absorption of solar radiation at the surface separately by the plants and by the soil - and treat separately the transfer of the absorbed radiation from these two surface components to the atmosphere or to the soil. Such calculations are presented here, based on a simple model previously developed by otterman (1981a). 
2. Absorption of solar radiation by the plants and by the soil

In this section we analyze how the combined absorption at the surface is increased by the protruding plants as a function of the time of day. In reading this and the following section, one can visualize the plants as floating at a height of several meters, rather than attached to the soil, and thus one can regard the absorption by the plants as equivalent to that by $a$ chaff layer. The model (Otterman, 1981a) represents the plants as thin vertical cylinders and the soil as a horizontal plane reflecting according to the Lambert law. The reflectance of the plant components is by a factor of more than 3 lower than that of the Sinai soil in the vicinity of the exclosure (Otterman, 1981b). We therefore make here a further simplifying assumption (as compared to the model presented in Otterman, 1981a) that the plants are total absorbers, of zero reflectance and zero transmittance. Based on these assumptions, the absorptivity $b_{d g}$ of the soil for the direct solar beam is:

$$
b_{d g}\left(s, r_{i}, \theta_{0}\right)=\left(1-r_{i}\right) \exp \left(-s \tan \theta_{0}\right),
$$

where $\theta_{0}$ is the solar zenith angle, $r_{i}$ is the Lambertian reflectance of the soil ( $i$ stands for interstices) and $s$ is the projection on a vertical plane of plants per unit area. Interception of solar radiation by the tree trunks was formulated in a similar way by Federer (1968) in his two-level forest canopy model. Our protrusion parameter $\mathbf{s}$ is identical to the silhouette parameter introduced by Lettau (1969) into formulation of surface roughness (Lettau denoted his silhouette parameter as $s / s$, where $s$ is the silhouette of an average obstacle and $S$ is the horizontal area it occupies). In Eq.(1), the term $\exp \left(-s \quad \tan \theta_{0}\right)$ denotes the fraction of the direct beam that penetrates through the plants and reaches the soil and thus $1-\exp \left(-s \quad \tan \theta_{0}\right)$ is the fraction intercepted and absorbed by the plants. The plants absorptivity $b_{d p}$ 
for the direct solar beam is:

$$
b_{d p}\left(s, r_{i}, \theta_{0}\right)=1-\exp \left(-s \tan \theta_{0}\right)+I(s) r_{i} \exp \left(-s \tan \theta_{0}\right),
$$

where the last term describes the absorption by the plants of the fractional flux $r_{i} \exp \left(-s \tan \theta_{0}\right)$ reflected from the soil. $I(s)$ is the fraction of that flux intercepted and absorbed by the plants:

$$
I(s)=2 \int_{0}^{\pi / 2} \cos \theta \sin \theta[1-\exp (-s \tan \theta)] d \theta,
$$

where $\theta$ is the zenith angle of a ray reflected from the soil.

The absorptivity of the surface $b_{d}$ for the direct beam (the co-albedo) is given by the sum of the plants absorptivity and the soil absorptivity:

$$
b_{d}\left(s, r_{i}, \theta_{0}\right)=1-r_{i}[1-I(s)] \exp \left(-s \tan \theta_{0}\right)
$$

The absorptivities $b_{d g^{\prime}} b_{d p}$ and $b_{d^{\prime}}$ and the ratio $P_{d}$ of the plant absorptivity to the combined surface absorptivity

$$
P_{d}\left(s, r_{i}, \theta_{0}\right)=b_{d p}\left(b_{d p}+b_{d g}\right)
$$

are now calculated, using 0.42 for the soil albedo $r_{i}$. This value for $r_{i}$ is based on the measurements in the northern Sinai and at the Sinai/Negev boundary from Landsat (Otterman and Robinove, 1982), and from the ground (Otterman, 1981b). For the protrusion parameter $s$ we adopt the value of 0.2 , as inferred for the 1974 Sinai exclosure from Landsat observations by Otterman and Robinove (1982) one and two years after the fencing off. The same value of $s$ was inferred by Otterman and Tucker (1985) from NOAA AVHRR data seven years after the fencing off. With these values of $r_{i}$ and $s$, we tabulate $b_{d g}{ }^{b} b_{d p} b_{d}$ and $P_{d}$ in the top four rows of Table 1 (below $\theta_{0}$ ) for solar 
zenith angles $30^{\circ}$ to $70^{\circ}$.

We note that the plant absorptivity $b_{d p}$ increases steeply with increasing $\theta_{0^{\prime}}$ while $b_{d g}$ decreases. Their sum, $b_{d^{\prime}}$ increases with $\theta_{0}$. The fraction $P_{d}$ increases steeply with $\theta_{0}$.

In the fifth row of Table 1 we tabulate the ratio $E_{\mathrm{da}}$ of the plants/soil surface absorptivity $b_{d}$ to that of the same soil bare of vegetation, that is, to the co-albedo of $1-r_{i}=0.58$. The ratios $b_{d} / 0.58$ increase from 1.23 at $30^{\circ}$ to 1.33 at $60^{\circ}$ and 1.40 at $70^{\circ}$. In these calculations solely for the direct solar beam, the dependence on the solar zenith angle $O_{O}$ is somewhat exaggerated, compared to the situation in which the scattered radiation is considered. The advantage of Eq. (1), (2) and (4) is their simplicity as compared to the equations for the global radiation (direct and diffuse) presented later. The vertical plants would enhance the surface absorptivity by somewhat higher ratios, if the anisotropy of the reflection from the soil (reflection at low elevation angles larger than that according to the Lambert law) is considered (Otterman et al., 1987).

A simplistic representation is made in this section that the nature of the plants/soil surface is (except for the lower albedo) identical to the bare soil surface. This would be the case if the dark plants would be embedded in the top layer of the soil, thus reducing the albedo without forming a nonplanar structure. Under this representation, the difference $R_{n}-G$ between the net radiation and the soil heat flux can have the same proportionality factor to $s_{n}$ the solar radiation absorbed at the surface, in the case of the vegetated (low-albedo) surface as in the case of the bare (high-albedo) surface. Idso et al. (1975), for instance, report the difference $R_{n}-G$ as a 
fraction of $s_{n}$. on the premise that for given wind conditions and thermal emission from the atmosphere $R_{n}-G$ is a specified fraction of $s_{n}$, the $b_{d} /\left(1-r_{i}\right)$ absorptivity (co-albedo) ratio represents the enhancement of the sensible (and latent, to the extent moisture is available) heat flux to the atmosphere.

We now repeat our calculations taking into account the scattered (diffuse) solar radiation. Because our surface model is azimuth independent, we have to consider only that the scattered radiation illuminates the surface generally at a different effective zenith angle than $\theta_{0}$. This effective zenith angle, denoted $\theta_{x^{\prime}}$ can be expected to be about $50^{\circ}$. It can be assumed that $\theta_{x}$ does not change when $\theta_{0}$ changes, if scattering by the aerosols into aureole region are excluded (The intense forward scattering by aerosols into the narrow cone around the direct beam can be simply added to the direct beam). The absorptivity $b_{x g}$ by the soil of the scattered radiation is thus

$$
b_{x g}\left(s, r_{i}, \theta_{x}\right)=\left(1-r_{i}\right) \exp \left(-s \tan \theta_{x}\right)
$$

and that by the plants

$$
b_{x p}\left(s, r_{i}, \theta_{x}\right)=1-\left[1-I(s) r_{i}\right] \quad \exp \left(-s \tan \theta_{x}\right)
$$

To assess the relative magnitude of the solar radiation absorbed at different solar zenith angles and different times of the day, we analyze the absorption rates of the solar radiation by the soil, $\mathrm{B}_{\mathrm{g}^{\prime}}$ and by the plants, $\mathrm{B}_{\mathrm{p}}$ (rather than the respective absorptivities). The solar radiation outside the atmosphere is given as $\cos \theta_{0}$. At the surface, the direct solar beam $f_{d}$ is given as

$$
f_{d}\left(\theta_{0}, T_{s}, T_{a}\right)=\cos \theta_{0} \exp f\left(T_{s}+T_{a}\right) / \cos \theta_{o} Z
$$


where $T_{S}$ is the scattering optical thickness and $T_{a}$ is the absorption optical thickness. The scattered irradiance flux $\mathrm{f}_{\mathrm{x}}$ is:

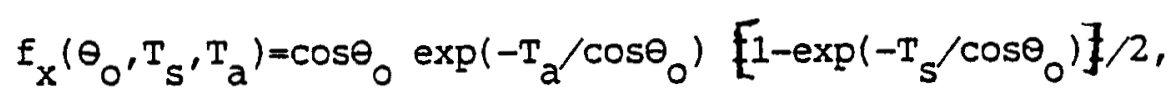

where the simplified single-scattering approach is used, which is a satisfactory approximation under low optical thickness conditions (Otterman, 1979), and where the intense scattering by aerosols into a narrow cone around the direct beam is added to the direct irradiation (scattering outside this cone is assumed to be equally divided between directions above and below the horizontal plane). The soil absorption rate $B_{g}$ is:

$$
B_{g}\left(s, r_{i}, o_{o}\right)=f_{d} b_{d g}+f_{x} b_{x g}
$$

and similarly the plant absorption rate $B_{p}$ is:

$$
B_{p}\left(s, r_{i}, o_{0}\right)=f_{d} b_{d p}+f_{x} b_{x p}
$$

Using the values $T_{a}=0.1$ and $T_{s}=0.1$, we compute $B_{p^{\prime}} B_{g^{\prime}}$ the ratio $P$ of the plants absorption rate to the surface absorption rate:

$$
P\left(s, r_{i}, \theta_{0}\right)=\frac{B_{p}}{B_{p}+B_{g}},
$$

and the enhancement $E_{a}$ of absorption by the plants/soil surface to that by the bare soil:

$$
E_{a}=\frac{B_{p}+B_{g}}{\left(E_{d}+f_{x}\right)\left(1-r_{i}\right)}
$$

These four functions are tabulated in the top rows of Table 2 (below $\theta_{0}$ ). We note first that at $\theta_{0}$ of $70^{\circ}, B_{p}$ is more than two thirds of $B_{p}$ at $30^{\circ}$, whereas $\mathrm{B}_{\mathrm{g}}$ at $70^{\circ}$ is only one fifth of $\mathrm{B}_{\mathrm{g}}$ at $30^{\circ}$. The surface absorption, the sum $\mathrm{B}_{\mathrm{g}}$ 
$+{ }_{p^{\prime}}$ is at $\theta_{0}=70^{\circ}$ about one third of the surface absorption at $30^{\circ}$. Comparing now $P$ and $E_{a}$ in Table 2 to $P_{d}$ and $E_{d a}$ in Table 1, we note that these ratios of absorption rates (or of absorptivities) are the same at $\theta_{0}$ of $50^{\circ}$, which is to be expected, inasmuch as we assumed $\theta_{x}$ of $50^{\circ}$. At solar zenith angles smaller than $50^{\circ}$, the ratios $\mathrm{P}$ and $\mathrm{E}_{\mathrm{a}}$ are higher than the ratios $\mathrm{P}_{\mathrm{d}}$ and $E_{\text {da }}$ for the direct beam only (the differences between the ratios $E_{d a}$ and $E_{a}$ for $\theta_{0}$ of 30,40 and $45^{\circ}$ show up only in the fourth decimal place); and conversely are lower when $\theta_{o}$ is larger than $50^{\circ}$. The enhancement $E_{a}$ at $70^{\circ}$ stands now as 1.38 , instead of 1.40 for the direct beam. In view of the many approximations used in these calculations, these numerical results suggest that under low levels of aerosols the calculations for the direct beam only are a satisfactory simpler substitute for the more accurate but more involved calculations for the global radiation.

The same functions are plotted in Fig. 1 vs. time of day $t$ in hours ( $t=6$ at sunrise, $t=12$ at noon). At equinox, the solar zenith angle $\theta_{0}$ is given as:

$$
\cos \theta_{0}=\cos [\pi(12-t) / 12] \cos L,
$$

where $\mathrm{L}$ is the latitude. $\mathrm{L}$ is $31.5^{\circ}$ at the Sinai/Negev boundary near the Mediterranean coast. The solar zenith angle, plotted in Fig. 1 (at the top), decreases from sunrise to the value of $31.5^{\circ}$ at noon. Both absorption rates increase in the morning but at different slopes: ${ }_{B_{p}}$ is larger than $B_{g}$ until about $0800 \mathrm{~h}$, but at noon $\mathrm{B}_{\mathrm{g}}$ is larger than $\mathrm{B}_{\mathrm{p}}$ by about 2.5 (see the middle graphs). The ratios $\mathrm{E}_{\mathrm{a}}$ and $\mathrm{P}$ decrease monotonically to noon (see the lower graphs). 
3. The second role of plants: the enhancement of the sensible heat flux to the atmosphere through the soil heat flux reduction.

Clothier et al. (1986) report that vegetation sharply reduces the soil heat flux. This reduction constitutes the second role of the plants: the heat not consumed by the day-to-night storage in the soil becomes available as a daytime heat input to the atmosphere. Assuming that the storage of heat in the dry, thin plants is negligible, we apply our model to formulate the reduced soil heat flux and the enhanced transfer of the absorbed solar radiation from the surface to the atmosphere as a function of time of day.

In the previous section we assumed that the $R_{\Omega}-G$ difference between the net radiation $R_{n}$ and the soil heat flux $G$ has the same proportionality factor to the solar radiation $s_{n}$ absorbed at the surface for both types of surface analyzed here. Thus, the ratio $\mathrm{E}_{\mathrm{a}}$ of the differences $\mathrm{R}_{\mathrm{n}}-\mathrm{G}$ for a low-albedo surface to a high-albedo surface is equal to the ratio of the respective absorptivities (co-albedo). We regard this assumption as simplistic because our vegetated terrain should be analyzed as a complex (nonplanar) surface. It can be reasonably assumed that the thin nearly vertical twigs and stalks are so thoroughly exposed to the air flow that the solar radiation they absorb is immediately released (as sensible heat flux) to the atmosphere. The intimate aerodynamic contact between the plants and the air flow is indicated by relatively small differences (when compared to the soil - air temperature differences) between the plants' radiation temperatures and the air temperatures, as reported in daylong measurements in the sinai exclosure (Otterman, 1981a).

Looking at these effects from a different viewpoint, the plants shadow the soil and thus reduce the solar radiation absorbed by the soil. The 
approach in this section to evaluating $R_{n}$ and $G$ is based on the assumption that the net radiation $R_{n}$ has the same proportionality factor to the solar radiation absorbed by the surface (that is, by the soil and by the plants, if any) in both the low-albedo (plants/soil) and the high-albedo (bare soil) case, whereas a different relation holds for $G$. The longwave radiation losses from the surface to the atmosphere are thus assumed to have the same proportionality factor to the absorbed solar radiation in the plants/soil case as in the bare soil case. In making this assumption, we err on the "conservative" side (we underestimate the sensible heat flux from the plants/soil surface), because actually the soil temperatures are reduced in the plants/soil surface as compared to the bare soil case (with the same solar radiation $S_{n}$ absorbed at the surface, and the plant temperatures are most of the day lower than these (reduced) soil temperatures. Moreover, the air temperatures over vegetated areas would tend to be higher due to reduced soil heat flux (see below), which would tend to reduce further the longwave radiation losses.

The soil heat flux $G$ is assumed to have the same proportionality factor in both cases to the solar radiation $s_{r}$ absorbed solely by the soil. $s_{r}$ equals $s_{n}$ in the case of bare soil, but in the case of plants/soil, $s_{I}$ is only a fraction of $s_{n}$ given by the ratio of the soil absorption rate to the total surface absorption rate:

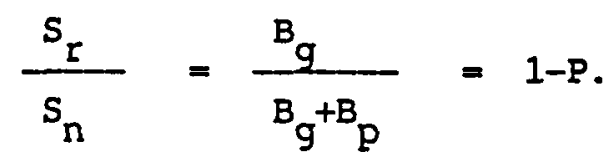

The symbols without the subscript $d$ indicate that the scattered solar radiation is considered. In terms of the absorptivities for the direct beam only, the expressions are simple: 


$$
\frac{s_{d r}}{s_{d n}}=\frac{b_{d g}}{b_{d g}+b_{d p}}=\frac{\left(1-r_{i}\right) \exp \left(-s \tan \theta_{0}\right)}{1-r_{i}[1-I(s)]} .
$$

In this formulation the influence of thermal emission from above on the soil temperatures, and thus on the soil heat flux, is not considered. This emission originates in part from the plants rather than from the atmosphere, (predominantly so at large viewing zenith angles). The plant temperatures are approximately equal to the air temperatures near the surface. At any usual air temperature profile this high emission would tend to increase $G$ as compared to the case of bare soil. The effect is small however, inasmuch as at large zenith angles the atmospheric emission originates anyway effectively from near-surface levels.

Clothier et al. (1986) relate through a series of measurements under various soil moisture conditions the relationship of $G$ to $R_{n}$. Their findings can be represented for our purpose as an approximate relationship:

$$
G=g_{0} R_{n}
$$

with the values of $g_{0}$ for dry soils of 0.4 or 0.5 . Similar measurements were. conducted in the Negev by Fuchs and Hadas (1972).

Let $E_{g}$ denote the enhancement by the plants of the sensible heat flux solely thorough the soil heat reduction (in addition to the enhancement $E_{a}$ through the albedo reduction). We can state that:

$$
E_{g}\left(s, r_{i}, g_{0}, \theta_{0}\right)=\frac{R_{n}-G_{r}}{R_{n}-G}=\frac{R_{n}\left(1-g_{r}\right)}{R_{n}\left(1-g_{0}\right)}
$$




$$
=\frac{1-g_{0}{ }_{q} /\left(B_{q}+B_{p}\right)}{1-g_{0}}=\frac{B_{g}+B_{p} /\left(1-g_{0}\right)}{B_{g}+B_{p}}=1+\frac{g_{0}}{1-g_{0}} P
$$

where $g_{r}$ is the ratio of the reduced soil heat flux $G_{r}$ in the plants/soil surface to $G$, the heat flux into the bare soil for the same absorption $S_{n}$ of solar radiation at the surface. These statements for $\mathrm{E}_{g}$ indicate that the absorption of solar radiation by the plants $B_{p}$ is by a factor $1 /\left(1-g_{0}\right)$ (that is, by a factor of 1.67 and 2.0 for $g_{0}$ of 0.4 and 0.5 respectively) more effective in producing sensible heat flux than the absorption $B_{g}$ by the soil. In Fig. 1, a scale is provided on the right, to assess $E_{g}$ from $P$ when $g_{0}=0.5$.

The enhancement ratio $E_{\mathrm{dg}}$ for the direct beam only (subscript d) is tabulated for $g_{0}=0.5$ and $g_{0}=0.4$ in rows 5 and 6 of Table 1 . The ratio $E_{g}$ for the global (direct and scattered) radiation is tabulated in the same rows of Table 2. We note that the enhancement ratio $E_{g}$ for $g_{0}=0.5$ is substantially higher than the enhancement $E_{a}$ by the reduction of the surface albedo. The combined enhancement ratios, the products $\mathrm{E}_{\mathrm{da}} \mathrm{E}_{\mathrm{dg}}$ (for the direct beam) and $E_{a} E_{g}$ (for the global radiation), are tabulated in the last two rows of Table 1 and Table 2, respectively.

Inasmuch as the second role of the plants, of reducing the day to night storage of heat at the surface, produces daytime effects higher than those solely by the albedo reduction (if $g_{0}$ is 0.5 ), the combined mesoscale effects can be more than double those hypothesized before (Otterman, 1974) on the basis of the albedo reduction alone.

There is an additional implication, for the need to support the albedo measurements by the surface temperature measurements. The transport of 
sensible heat from the surface to the atmosphere is proportional to the product of the temperature gradient above the surface by the turbulent transfer coefficient for heat, see for instance Eq. (11.9) in Hillel (1980). If it is assumed that the aerodynamic contact of the low-albedo surface with the air flow is the same (the same turbulent transfer coefficient for heat) as that of the high-albedo surface, the increased sensible heat transfer to the atmosphere for the low-albedo surface should be evidenced by higher surfaceto-air temperature differences (if both surfaces are dry). That means higher surface temperatures if air temperatures are the same. When early Landsat images showed in the spectral bands of the Multispectral Scanner System a high spectral albedo of the overgrazed Sinai vs a much darker Negev, this consideration prompted an instrumented aircraft flight back and forth across the Sinai/Negev boundary. The August 31, 1973, $1400 \mathrm{~h}$ airborne measurements in the broad 8-11 $\mu \mathrm{m}$ thermal band showed the radiation temperatures of the vegetated Negev to be 4 to $5 \mathrm{k}$ higher than those of the overgrazed sinai (in the area of the highest contrast, where there was a fence at the boundary). Because of the narrow field of view of the PRT-5 radiometer and the vertical structure of the vegetation, in the Negev the instrument views predominantly the interstices of soil between the plants. (our model, which predicts that plants are not visible when viewing exactly from zenith, should not be applied here). From these measurements, the rainfall reduction mesoscale effects of overgrazing were formulated (Otterman, 1974) to parallel the study of Black and Tarmy (1963), who suggested tarring the Mediterranean coastal areas to increase the surface daytime temperatures and thus increase convection and precipitation.

The interpretation of these radiation temperature measurements in the broad thermal band, which included the restrahlen band, was faulted for not 
taking into account the reduced emissivity of sand in the restrahlen band (Jackson and Idso, 1975). Measurements of radiation temperatures at $11.5 \mu \mathrm{m}$, outside the restrahlen band, from NOAA AVHRR (early morning passes) always showed higher radiation temperatures in the vegetated exclosure than in the surrounding overgrazed terrain. The differences ranged from $0.1 \mathrm{~K}$ to $2.9 \mathrm{~K}$. No correlation could be found with wind speed or time from sunrise, to explain the spread in these temperature differences (Otterman and Tucker, 1985). Thus, the temperature measurements do suggest a higher surface-to-air gradients over desert-fringe vegetated area than over adjacent bare soil. However, these higher temperatures and higher gradients are not required as evidence of the higher sensible heat flow from the surface if the plants facilitate the heat transfer to the air flow as compared to that from the bare soil. This facilitated transfer can be readily inferred by considering that in our model the area of contact with the airflow of plants per unit surface area is $\pi \mathrm{s}$. Thus, in the plants/soil case with $s=0.2$, the combined area of contact (of soil and of plants) is larger by a factor 1.63 than in the case of the planar surface of soil without plants. 


\section{Discussion and conclusions}

our analysis suggests that reduction of the soil heat flux can sharply enhance the daytime sensible heat flux to the atmosphere. The enhancement is especially strong at large solar zenith angles. For the high soil albedo measured in the Sinai/Negev from the ground (Otterman, 1981b) and from Landsat (Otterman and Robinove, 1982), and the high value of the soil heat flux, $G=$ $0.5 R_{n}$, taken from the measurements of Clothier et al. (1986), the enhancement is by a factor of 1.6 at large solar zenith angles and by 1.3 at moderate zenith angles. It is thus significantly larger than the enhancement by the surface albedo reduction. The time-of-day representation of the reduced soil heat flux adopted here has not been demonstrated experimentally-it is only a plausible application of our model (Otterman, 1981a). The substantial magnitude of the reduction of the soil heat flux by plants has been firmly established by measurements of Clothier et al. (1987). They report $G / R_{n}$ ratios in the 0.4 to 0.5 range for bare dry soil, and about 0.15 for dry vegetated terrain. These results can be translated (without using our model) into enhancement of the $R_{n}-G$ differences by factors of 1.4 to 1.7 . Even the highest ratios $\mathrm{E}_{\mathrm{g}}$ that we present (at solar zenith angle of $70^{\circ}$, see Table 2 ), are lower than that. It would be very interesting to conduct the measurements of soil heat flux for vegetated and bare surfaces in desert-fringe regions.

The combined effects of the albedo and soil heat flux reduction are about 2 at large zenith angles. Such effects can be expected to cause a significant increase in the daytime growth of the planetary boundary layer (PBL). A study by Berkofsky (1977), in which the vertical velocities at the top of the boundary layer are related to the albedo and the sensible heat flux, is pertinent to the problem area. We do not directly address the PBL growth, 
which should be studied by daylong simulation of the surface-atmosphere interactions. Such a simulation is currently being attempted (Otterman and McCumber, in preparation).

We assume the $R_{n} / S_{n}$ ratio to be the same for the plants/soil surface as for the bare soil. In reality the ratio for the plants/soil should be appreciably higher, because the longwave loss from the surface to the atmosphere is lower: in the plants/soil surface the soil temperatures are reduced as compared to bare soil case (for the same $s_{n}$ ) and the plant temperatures are most of the day significantly lower (closer to the air temperatures) than these reduced soil temperatures. This assumption leads to underestimating the enhancement of the sensible heat flux by the plants. However, it should be recognized that a significant fraction of the longwave loss from the surface is absorbed within the boundary layer, and this heating can produce the same effects as the sensible heat flux.

We do not analyze here how the atmospheric temperatures rise as a consequence of the higher sensible heat flux due to the reduced soil heat flux. A rise in the atmospheric temperatures would change the thermal radiation exchange between the surface and the atmosphere, and this in turn would affect the soil heat flux and the sensible heat flux. Without considering such interactions, the enhancement ratios that we calculate should be considered as only preliminary, with fuller evaluation to be obtained in daylong simulation.

The difficult question is, how the enhancement of the daytime sensible heat flux affects the cloud formation and the probabilities of convective precipitation. Our discussion is certainly not quantitative in terms of 
rainfall. The analysis of Black and Tarmy (1963), in which elevated surface temperatures of a tarred (low-albedo) surface are related to a large increase in the precipitation, might not be quantitatively appropriate (Otterman, 1974). A careful statement by Malkus (Joanne Simpson) is appropriate here: "there exists at least indirect evidence that useful water might be obtained in some localities from increased cumulus showers in weakly disturbed or undisturbed conditions where skies are generally sunny enough for a thermal mountain to be effective" (Malkus, 1963).

Our analysis deals with mesoscale phenomena, and we do not address the large scale effects of regional high albedo, that produce descending airmasses over the desert, as analyzed by Charney (1975). Such descending airmasses and the associated inversions are important characteristics of the climate in Israel and the Sinai throughout the sumer, see for instance shaia and Jaffe (1976). The albedo effects accrue in the daytime only, but can be integrated over a day or over several days. The soil heat reduction effects accrue also in the daytime. However, for this effect the integration over 24 hours produces practically a null difference in the sensible heat flux. In other words, if heating from the plants/soil surface does not produce squall lines by evening, the effect of the reduced soil heat flux is null because the reduced heat flux from such a surface at nighttime (as compared to that from bare soil) would tend to produce at sunrise an atmospheric profile less conductive to cloud formation. (Likely non-linearities of the boundary layer growth in terms of the sensible heat flux make this statement only approximately true).

The effects of soil heat flux reduction on convection and convective precipitation is limited to daytime, and can be expected to be especially strong in the morning and afternoon hours, at large solar zenith angles. The 
precipitation in the arid regions is indeed a predominantly intense convective daytime phenomenon and this predominance increases with increased aridity (Sharon, 1972; Kutiel, 1977). In the western and northern Negev the day/night partitioning is somewhat complicated: the early rains, October - November, show strong daytime predominance, while the opposite is true for the later rains (Otterman and Sharon, 1979). The interesting question in the case of the African drought is, at what time of day should the rains that failed to materialize in a drought year, have occurred? A study of the diurnal variations in convective activity and precipitation during GATE (McGarry and Reed, 1978) indicates that in the sub-Sahara region much of the rainfall is produced by squall lines that typically form in the afternoon as a result of the daily heating cycle.

Our assessment of the effects of vegetation (or the consequences of its removal) is believed to apply quantitatively to the Sinai/Negev region, where the soil albedo is high, where overgrazing results in an essentially bare soil, and where outside of the desert bloom period the vegetation contains very little moisture. These dry twigs and stalks are more like stubble than like the green vegetation in more humid regions. Our calculations should not be quantitatively applied to all arid regions. In the Kalahari and in northern Mexico, bushes or trees remain in the overgrazed area. The effects of overgrazing must be much weaker there, both in terms of the albedo and of the soil heat reduction. The trees and bushes in such less arid (compared to Sinai/Negev) areas do contain appreciable moisture, and the daytime heat storage in these plants cannot be regarded as negligible. Finally, the effects would be smaller in a region where soils have an albedo lower than the 0.42 found in Sinai/Negev. 
The Sinai/Negev vegetation is not unique, however. The desert shrub rangelands in Iraq exhibit the same vertical structure in clumps of thin twigs and stalks, dark against the background of bright soil, as shown in numerous photographs presented by Thalen (1979). Thalen also reports the rapid and dramatic recovery of vegetation in overgrazed land, simply by forming an exclosure. This confirms our findings (Otterman, 1981b; Otterman and Robinove, 1982) and our opinion, that the vegetative cover (outside the desert bloom period, during the rainy season) is primarily a function of the grazing practices, and not of past precipitation.

It is thus an open question to what extent our analysis of possible mesoscale effects of overgrazing applies to the problem of African drought. Applicability of our calculations can be advocated most strongly for the extremely arid regions. Under conditions of extreme aridity, the "mean" rainfall does not convey the same meaning as in more humid regions, because of the large year-to-year fluctuations. We should regard precipitation in terms of probabilities of zero, one, two or three rain events in a year. In such regions, the current practice in many African countries of maintaining a large population of grazing animals results in reduced vegetation density. The reduced vegetation, through the mesoscale mechanism described here, can reduce convective precipitation, inducing and perpetuating higher probabilities of drought. The drought perpetuating influence is a recent development. In the past, the grazing animals would die off or be slaughtered in a drought. The pressure on vegetation would be reduced. Today support in fodder and water comes in a drought event, and vegetation continues to be under unrelenting impact, that is not alleviated by previous checks and balances. 


\section{Acknowledgments}

This study was supported in part by NASA contracts NAS5-28085 with Science Systems and Applications, Inc., Seabrook, MD and NAG5-861 with the University of Arizona, Tuscon, Arizona. Helpful comments by R. Kandel, Laboratoire de Meteorologie Dynamique, Palaiseau, France, and by M. McCumber, J. Susskind and T. Brakke, NASA/GSFC, Greenbelt, MD USA, who also provided help in the calculations, are gratefully acknowledged.

\section{Figure Captions}

Figure 1 The solar zenith angle $o_{0}$ (the top graph), the absorption rate $B_{g}$ by the soil and the absorption rate $B_{p}$ by the plants (in the middle), the enhancement ratio $\mathrm{E}_{\mathrm{a}}$ of absorption by plants/soil surface to that by bare soil and ratio $\mathrm{P}=\mathrm{B}_{\mathrm{p}} /\left(\mathrm{B}_{\mathrm{p}}+\mathrm{B}_{\mathrm{g}}\right)$ (the lower graphs), all plotted vs time of day from sunrise $(600 \mathrm{~h})$ to noon $(1200 \mathrm{~h})$ at equinox, at the Sinai/Negev boundary. 
TABLE 1. The soil absorptivity $b_{d g^{\prime}}$ the plant absorptivity $b_{d p^{\prime}}$ the plant absorptivity as a fraction $\mathrm{P}_{\mathrm{d}}$ of the surface absorptivity, the enhancement factor $E_{\mathrm{da}}$ by albedo reduction, the enhancement factor $E_{\mathrm{dg}}$ by soil heat flux reduction (for $g_{0}$ of 0.5 and 0.4 ) and the combined enhancement factor $E_{d}=$ $E_{\mathrm{da}} \cdot E_{\mathrm{dg}}$ (for $g_{0}$ of 0.5 and 0.4 ), all calculated for the direct solar beam only at different solar zenith angles.

$\theta_{0}$ (degrees)

$b_{d g}$

$b_{d p}$

$b_{d}$

$P_{d}$

$E_{\mathrm{da}}=\frac{b_{d g}+b_{d p}}{0.58}$

$E_{d g}=1+\frac{g_{0} P_{d}}{1-g_{0}}$

$E_{d a ̀} E_{d g}$
30

$0.517 \quad 0.490$

40

45

50

60

70

0.475

0.457

0.410

0.335

0.194

0.235

0.259

0.287

0.360

0.478

0.711

0.726

0.734

0.744

0.770

0.813

0.273

0.324

0.353

0.386

$0.468 \quad 0.588$

1.23

1.25

1.27

1.28

1.33

1.40

$$
g_{0}=0.5
$$

1.27

1.32

1.35

1.39

1.47

1.59

$$
g_{0}=0.4
$$

1.18

1.22

1.24

1.26

1.31

1.39

$$
g_{0}=0.5
$$

1.56

1.66

1.71

1.78

1.95

2.22

$\begin{array}{lllllll}g_{0}=0.4 & 1.45 & 1.52 & 1.56 & 1.61 & 1.74 & 1.95\end{array}$


TABLE 2. The absorption rate $B_{g}$ by the soil, the absorption rate $B_{p}$ by the plants, the ratio $P=B_{p}\left(B_{P}+B_{g}\right)$, the enhancement factor $E_{a}$ by albedo reduction $E_{a}=\left(b_{p}+b_{g}\right) / 0.58$, the enhancement factor $E_{g}$ by soil heat $f l u x$ reduction (for $g_{O}$ of 0.5 and 0.4 ) and the combined enhancement factor $E=E_{a} E_{g}$ (for $g_{0}$ of 0.5 and 0.4 ), all calculated for the global irradiance at different solar zenith angles.
$\theta_{0}$ (degrees)
30
40
$45 \quad 50$
$60 \quad 70$
Bg
$\begin{array}{llllllll}0.3745 & 0.3081 & 0.2716 & 0.2333 & 0.1544 & 0.0786\end{array}$
${ }_{\mathrm{p}}$
$\begin{array}{lllllll}0.1454 & 0.1505 & 0.1498 & 0.1466 & 0.1314 & 0.1004\end{array}$
$\mathbf{P}$
$\begin{array}{llllll}0.280 & 0.328 & 0.356 & 0.386 & 0.460 & 0.561\end{array}$
Ea
$\begin{array}{llllll}1.23 & 1.25 & 1.27 & 1.28 & 1.32 & 1.38\end{array}$
$E_{g}=1+\frac{g_{0}}{1+g_{0}}$

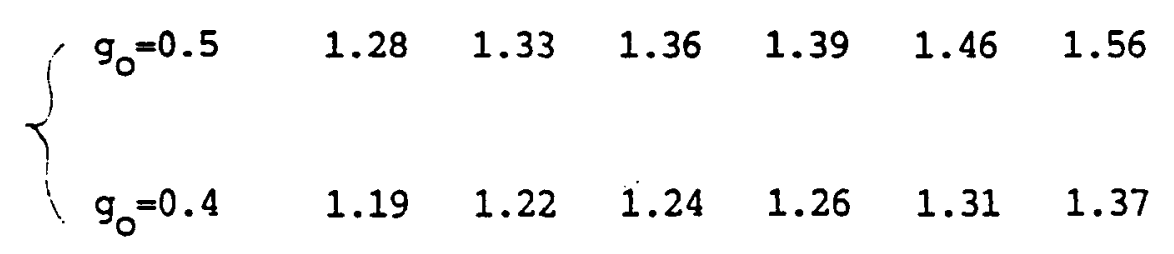
$E=E_{\dot{a}} E_{g} \quad\left\{\begin{array}{lllllll}g_{0}=0.5 & 1.57 & 1.66 & 1.72 & 1.78 & 1.93 & 2.16 \\ g_{0}=0.4 & 1.46 & 1.57 & 1.61 & 1.61 & 1.73 & 1.90\end{array}\right.$ 


\section{REFERENCES}

Berkofsky, L. 1977, The relation between surface albedo and vertical velocity in a desert, Beitrage zur Physik der Atmosphare 50, 312-320.

Black, J.F., and Tarmy, B.L: 1963, The use of asphalt coatings to increase rainfall, J. Appl. Meteor. 2, 557-564.

Charney, J. G.: 1975, Dynamics of deserts and drought in the Sahel, guart. J. Meteorol. Soc. 101, 193-202.

Clothier, B.E., Clawson, K.L., Pinter Jr., P.J., Moran, M.S., Reginato, R.J., and Jackson, R.D.,: 1986, Estimation of soil heat flux from net radiation during the growth of alfalfa, Agricultural and Forest Meteorology 37, 319-329.

Federer, C.A.: 1968, Spatial variation of net radiation, albedo and surface temperature of forests, J. of Appl. Meteor. 7, 789-795.

Fuchs, M., and Hadas, A: 1972, The heat flux density in a non-homogeneous bare loessial soil, Boundary Layer Meteorol. 3, 191-200.

Hillel, D.: 1980, The Fundamentals of Soil Physics, Academic Press, New York.

Idso, S.B., Aase, J.K., and Jackson, R.D: 1975. Net radiation - soil heat flux relations as influenced by soil water content variations, Boundary Layer Meteor. 9, 113-122.

Jackson, R.D., and Idso, S.B.: 1975, Surface albedo and desertification, Science 189, 1012-1013.

Kutiel, H.: 1977, The distribution of rainfall by intensity in Israel (in Hebrew). M. Sc. Thesis, Department of Geography, Hebrew University Jerusalem, 
Israel.

Lettau, H.H.: 1969, Note on aerodynamic roughness - parameter estimation on the basis of roughness - element description, J. of Appl. Meteor. 8, 828-832.

Malkus, J.S.:1963, Tropical rain induced by small natural heat source, J. Appl. Meteor. 2, 547-556.

MCGarry, M.M., and Reed, R.J.: 1978, Diurnal variations in convective activity and precipitation during Phases II and III of GATE, Monthly Weather Review $106,101-113$.

Nicholson, S.E.: 1979 Climatic variations in the Sahel and other African regions during the past five centuries, J. of Arid Environments 1, 3-24.

Otterman, J.: 1974, Baring high - albedo soils by overgrazing: a hypothesized desertification mechanism, Science 186, 531-533.

Otterman, J.:1978, Single - scattering solution for radiative transfer through a turbid atmosphere, Appl. Opt. 17, 3431-3438.

Otterman, J.: 1981a, Plane with protrusions as an atmospheric boundary, J. Geophys. Res 86, 6627-6630.

Otterman, J.: 1981b, Satellite and field studies of man's impact on the surface of arid regions, Tellus 33, 68-77.

Otterman, J., and Sharon, D.: 1979, Day/night partitioning of rain in an arid region: computational approaches, results for the Negev, and meteorological/climatological implications, J. Rech. Atmos. 13, 11-20. 
Otterman, J., and Robinove, C.J.: 1982, Landsat monitoring of desert vegetation growth, 1972-1979, using a plant shadowing model, Advances in Space

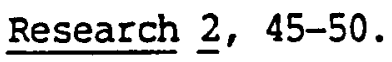

Otterman, J., and Tucker, C.J.: 1985, Satellite measurements of surface albedo and temperatures in semi desert, J. Climate and Appl. Meteor. 24, 228-235.

Otterman, J., Deering, D., Eck, T., and Ringrose, S.: 1987, Techniques of ground-truth measurements of desert-scrub structure, Advances in space Research, in press.

Shaia, J.S, and Jaffe, S.: 1976, Midday inversions over Bet Bagan, Series A Meteorological Notes \#33, Israel Meteorological Service, Bet Dagan, Israel.

Sharon, D.:1972, The spottines of rainfall in a desert area. J. Hydrol. I. 161-175.

Thalen, D.C.P.: 1979, Ecology and utilization of desert shrub rangelands in Iraq, Dr. W. Junk B. V. Publishers, The Hague.

Waisel, Y.: 1986, Interactions among plants, man and climate: historical evidence from Israel, Proceedings of the Royal Society of Edinburgh 89B, 255264. 

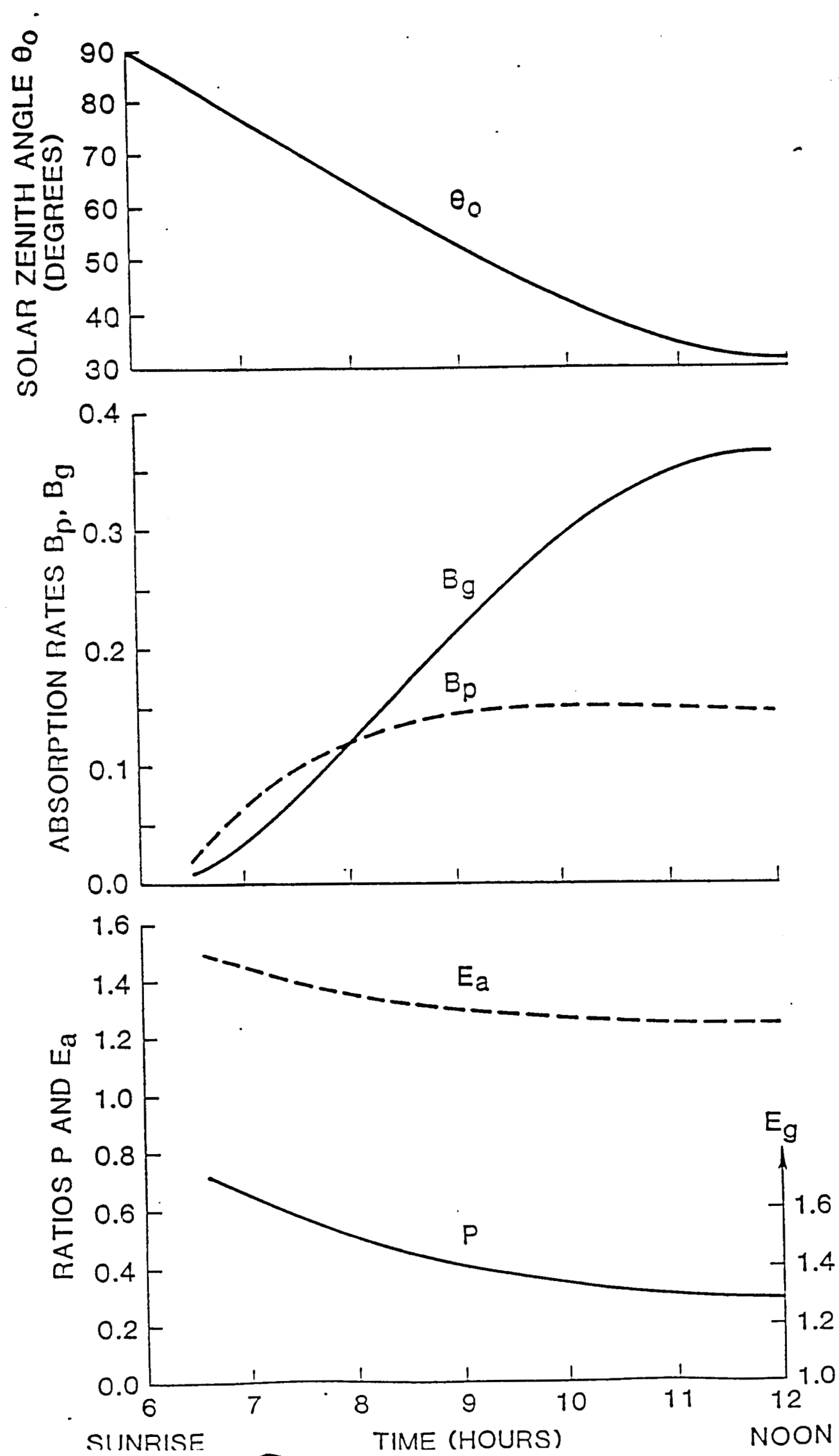